\title{
DIGITAL ELEVATION MODEL TO VISUALIZE SMALL SPATIAL OBJECT IN 3D
}

\author{
Cahyo Crysdian \\ Teknik Informatika,UIN MALIKI Malang \\ Jl. Gajayana 50 Malang \\ Telp. 0816-4299881, Email: crysdian@yahoo.com
}

\begin{abstract}
An effort to develop a Digital Elevation Model (DEM) for small scale spatial objects is presented in this paper. The motivation of the research is to visualize a small spatial object as detail as possible in 3D, thus the presentation of the object in term of a computer model is similar to its presentation in reality. To reach this objective, the research was conducted in four stages i.e. elevation data retrieval, $3 D$ visualization and its enhancement, and the development of blank data removal. Elevation data is obtained from SRTM dataset that has 3 arc-second or approximately 90 meters data resolution. Data obtained from SRTM is then visualized in 3D, in which visualization is enriched with view angle setting. Results of visualization show that enhancement to SRTM data set is required to present elevation data in 3D. It is due to the existence of blank data contained in the elevation data retrieved from SRTM. Therefore, this paper proposes two methods to enhance SRTM dataset i.e. population average and neighbors average, in order to counter the existence of blank data. The first method uses the population average of statistic to eliminate blank data, while the latter relies on the average value of its neighboring pixels. Comparison among those methods is held in this research to disclose the performance of each method. Result of comparison shows that the method based on neighbors average outperform population average method to eliminate blank data contained in SRTM data set. Thus, neighbor average delivers better $3 D$ visualization for small scale spatial object.
\end{abstract}

Keywords: Digital Elevation Model, 3D Visualization, Spatial

\section{INTRODUCTION}

The importance of spatial data to enrich the information conveyed to the users has been admitted by many researchers $[1,2,3]$. Some implementations to mention such as Yahoo Maps, Singapore online maps, Google Earth have presented the power of spatial data. However, 2D presentations of this data could not fulfill user satisfaction [1]. Users demand more realistic information using 3D visualization. This phenomenon raises the need for Digital Elevation Model (DEM) that deliver 3D visualization of spatial objects. The height of an object becomes the object of interest in these efforts. In case of spatial object, the elevation data is required to present the height of an object.

Literatures disclose the works on DEM were accomplished using three different approaches to obtain elevation data i.e. ground survey using GPS and other terrestrial equipments [2], unmanned flying vehicle $[3,4]$, and satellite-based DEM [5,6]. The first two-methods cover only small region of the earth although detail elevation data could be retrieved. While the last cover larger region since elevation data is collected by satellite. However, it is not without any drawbacks since satellite-based DEM produces difference accuracy for different regions 
[5]. Moreover, it is significantly influenced by climate condition and carries coarse elevation data $[6,7]$.

The goal of this research is to have detail visualization of small spatial objects such as a volcano or mountain. Since any specific sources of elevation data carry their own drawback as explained above, combination among different type of elevation data is desired. Thus, the availability of data sources plays a crucial role to accomplish the research.

\section{ELEVATION DATA MODEL}

Shuttle Radar Topographic Mission (SRTM) data set from US Geological Survey is used to construct an elevation data model. SRTM which is composed by a 3 arc-second or approximately 90 meters grid of elevation is a global elevation data collected by a space shuttle mission [7]. The data covers $80 \%$ of the earth surface spanning from 60 degrees north latitude to 54 degrees south latitude.

The elevation data source as explained above is read using a $2 \mathrm{D}$ matrix that consists of row and column. In this approach, row and column of the matrix represent longitude and latitude of the coordinate system respectively, while the elevation value is stored in each cell of the matrix. It is shown in Figure 1. The elevation data would consume a big size of memory if it is read from the data source entirely at once, thus storing the elevation data into the 2D matrix is limited only to the region of interest. With this strategy the performance of the machine can be maintained since the matrix to hold elevation data has a small size.

To implement the mechanism explained above, a specific location, i.e. Malang city which is located at $112^{\circ} 06^{\prime}-112^{\circ} 07^{\prime}$ east longitude and $7^{\circ} 06^{\prime}-8^{\circ} 02^{\prime}$ south latitude [9], is used to develop elevation data model. The consideration is the condition of Malang city which is geographically attractive since it is surrounded by several mountains.

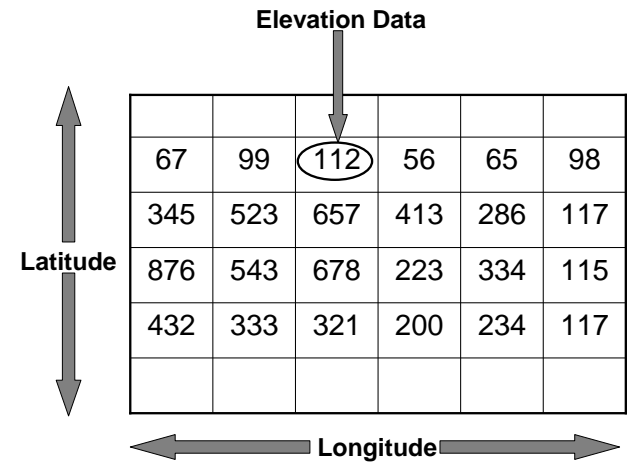

Figure 1. Matrix to store elevation data

\section{3D VISUALIZATION}

After reading elevation data to a $2 \mathrm{D}$ matrix, 3D visualization is developed. Transformation from 2D matrix to $3 \mathrm{D}$ visualization is done by converting row, column, and content of each cell to visualization coordinate represented by a set of axis namely $\mathrm{x}, \mathrm{y}$, and $\mathrm{z}$-axis respectively. This approach make the row of matrix represents the X-axis, the column represents the $y$-axis, while the content of each cell represents the value of $\mathrm{z}$-axis. Results of visualizations are shown in Figure 2.

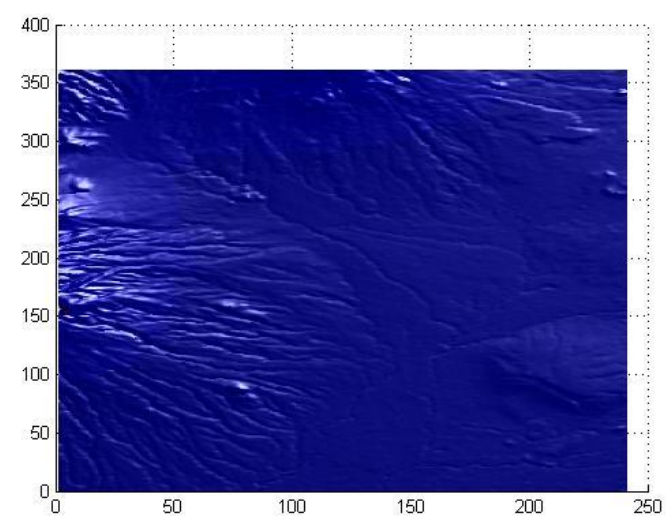

Figure 2. Results of visualization

To have better visualization, the view angle is developed as formulated below:

$$
V=(h, v)
$$


Which $V$ is the view angle, $h$ is horizontal elevation from y-axis, and $v$ is vertical elevation from $x-y$ plane as shown in Figure 3. Results of applying view angle to the visualized spatial objects are shown in Figure 4.

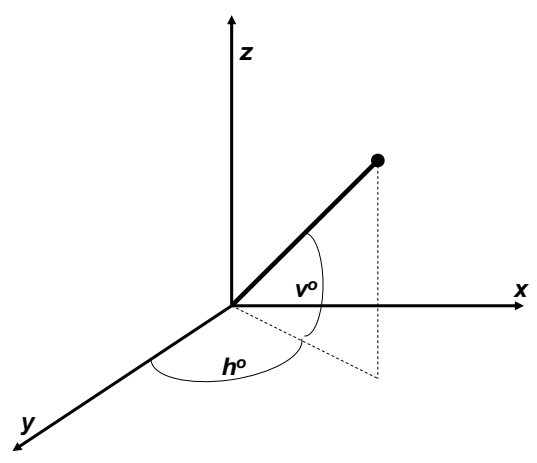

Figure 3. View angle

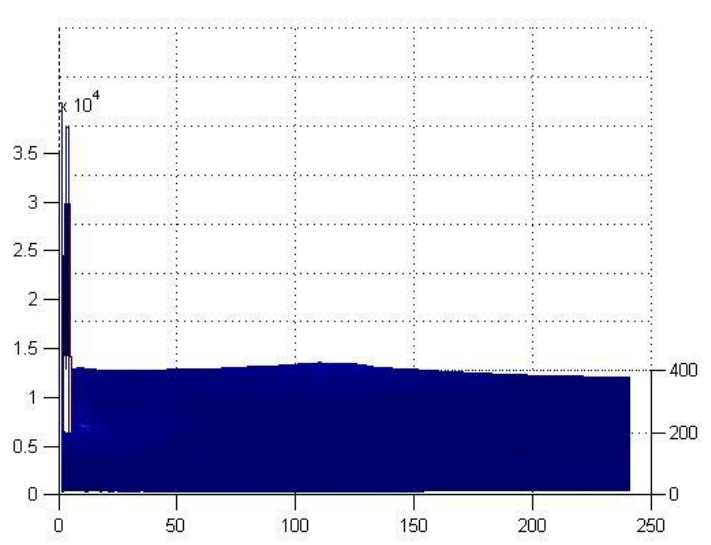

a. View angle $(0,20)$

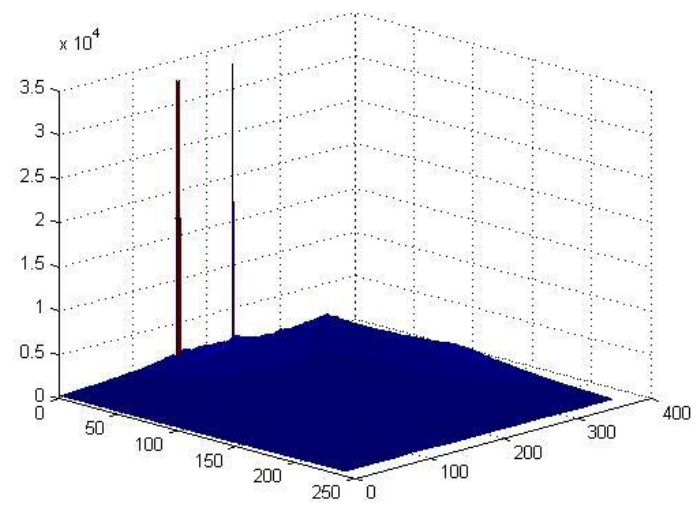

b. View angle $(45,20)$

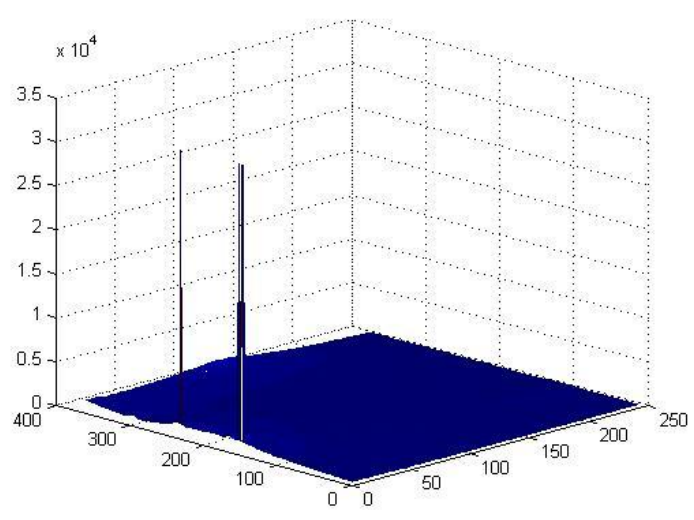

c. View angle $(-45,20)$

Figure 4. 3D Visualization using different setting of view angles

\section{ENHANCED VISUALIZATION BASED ON POPULATION AVERAGE}

Results of 3D visualization produced from the previous section as shown in Figure 4 disclose unbalanced correlation between horizontal coordinate represented by $x-y$ plane to vertical coordinate represented by z-axis. It seems that vertical data dominates horizontal value. This phenomenon is due to the existence of blank data obtained from elevation data source that causes improper visualization of spatial object such as noted by [8].

To solve this problem, a mechanism to fill up blank data and replace them with a specific data value is developed. This method employs a statistical approach based on population average. It is formulated as follows:

$$
A=\frac{\sum_{i=0}^{r m(M)} \sum_{j=0}^{c m(M)} d_{i, j}}{n} \leftrightarrow d_{i, j} \neq b
$$

With $A$ is a population average value that is used to replace blank data, $n$ is the number of times $d$ being added as a population member, and $\mathrm{rm}(\mathrm{)})$ and $\mathrm{cm}(\mathrm{)})$ is the function to determine the maximum value of row and column respectively from 
a matrix $M$ which holds elevation data value. Results produced by this method is given in Figure 5. Meanwhile Figure 6a to $6 \mathrm{c}$ show visualization of spatial object as presented in Figure 5 with different view angles similar to Figure $4 \mathrm{a}$ to $4 \mathrm{c}$ respectively.

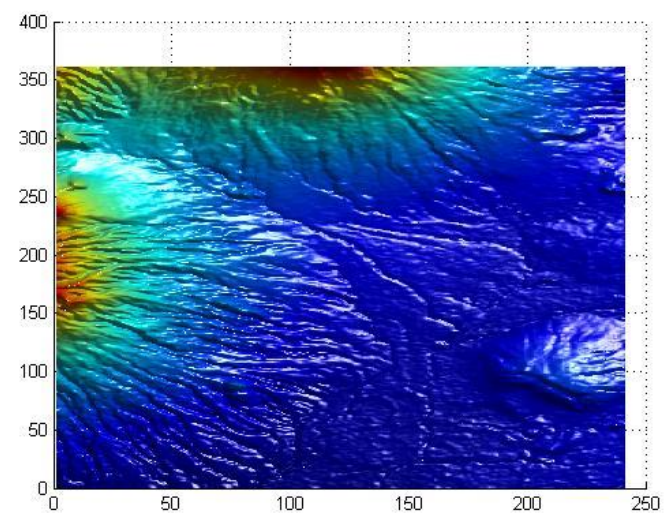

Figure 5. Results of enhanced visualization based on population average

Compared to Figure 2, Figure 5 presents better performance to visualize the location of interest. Contour of spatial objects is clearly shown in this figure. Moreover, viewing Figure 5 from different view angle as given in Figure $6 \mathrm{a}$ and $6 \mathrm{~b}$, with view angle is set to $(0,20)$ and $(45,20)$ respectively, show successful application of population average to replace blank data.

However, if view angle is set to (45,20 ) as shown in Figure 6c, population average produces several abnormal values. These values refer to the elevation data that differs with the surrounded elevation value. This problem is appeared due to the mechanism of population average to forcedly replace blank data with a specific value obtained from Equation 1 regardless its neighbor's value. Thus, the performance of this method is deteriorated by its own mechanism to replace blank data.

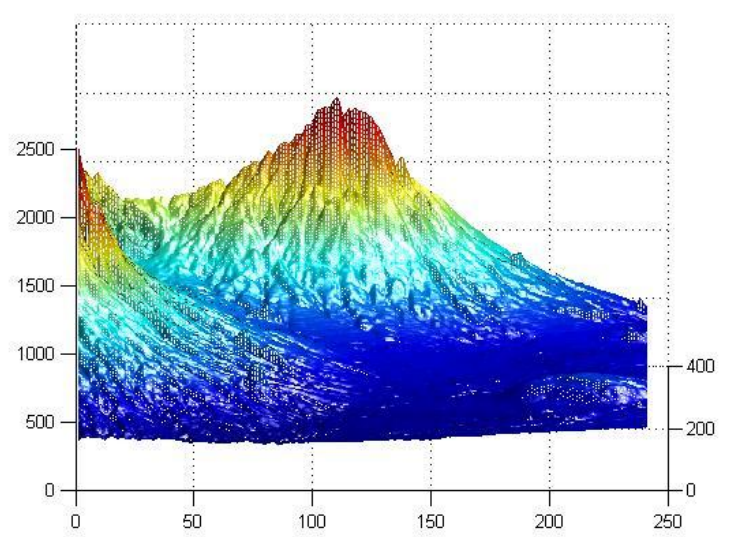

a. View angle $(0,20)$

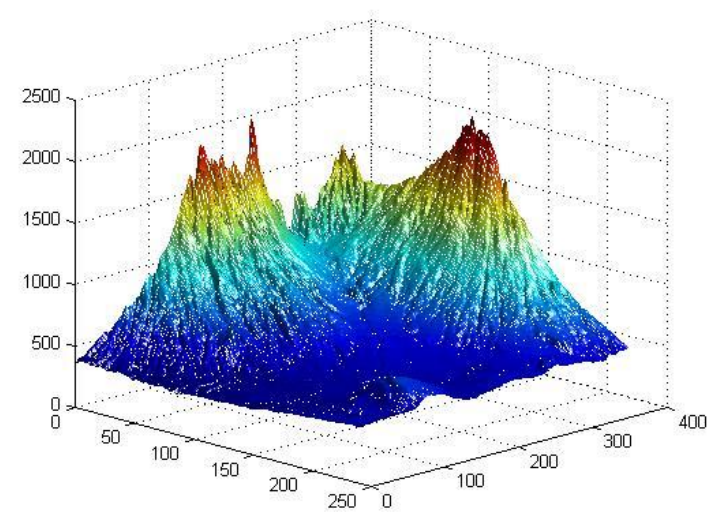

b. View angle $(45,20)$

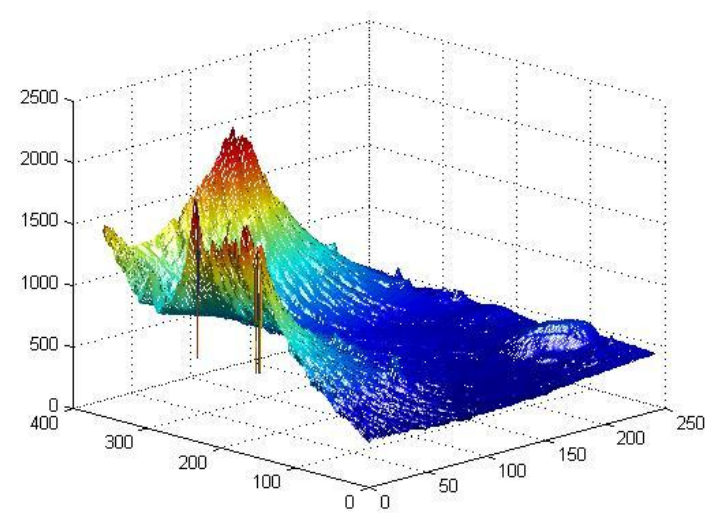

c. View angle $(-45,20)$

Figure 6. Enhanced visualization using different view angles

\section{NEIGHBORS AVERAGE}

Problem of population average to replace blank data as explained in the previous section is the absence of knowledge of its neighbors value. Therefore it is necessary to develop a mechanism to recognize the neighborhood 
of blank data in order to determine the elevation value of blank data. It is formulated as follows. Let $M$ becomes a matrix of elevation data with $i$ and $j$ become the index of row and column of $M$ respectively, and $d$ is the elevation data contained in $M$, a blank data $b$ at coordinate $(i, j)$ is replaced with the following value:

$$
b_{i, j}=\frac{d_{i-1, j}+d_{i+1, j}+d_{i, j-1}+d_{i, j+1}}{4}
$$

Equation 2 is applicable if only if $d_{i+1, j} \neq b$ and $d_{i, j+1} \neq b$. If either one or both value of $d_{i+1, j}$ or $d_{i, j+1}$ is a blank data, then the value of $d_{i+1, j}$ or $d_{i, j+1}$ is replaced with $d_{i-1, j}$ or $d_{i, j-1}$ prior to run Equation 2 to a matrix of elevation data. This approach avoids blank data to compute Equation 2 . Results produced by this method are presented in Figure 5.

Comparing Figure 6 and 7 as the product of population average and neighbor average respectively is done to each view angle setting used in this research. The objective is to evaluate the performance of each method. For view angle $(0,20)$ and $(45,20)$, both visualization delivered by population and neighbor average are similar as shown in Figure 6a and $6 \mathrm{~b}$ for population average, and Figure $7 \mathrm{a}$ and $7 \mathrm{~b}$ for neighbor average. However, the difference of both method is appeared when view angle is set to $(-45,20)$ as shown in Figure 6c for population average and Figure $7 \mathrm{c}$ for neighbor average. Unlike Figure $6 c$ that suffers from several abnormal values, visualization produced by neighbor average is capable to avoid the appearance of abnormal values as shown in Figure 6c.

\section{CONCLUSIONS}

Development of digital elevation model for small scale spatial objects is presented in this paper. The research comprises four stages of work as follows, the development of elevation data, 3D visualization and adjustment of view angle setting, enhanced visualization using population and neighbors average, and the last is comparison among the presented methods. The experiment shows that legacy elevation data retrieved from SRTM data set contain blank data values that prevent $3 \mathrm{D}$ visualization. It is due to the value of blank data which is too great compared to normal elevation data. Thus, two methods are presented to replace blank data i.e. population average and neighbors average.

Experiment proved that both population and neighbor average successfully replaced blank data values and enhanced 3D visualization. Better digital elevation models are delivered by both methods compared to legacy elevation data. However for specific view angle setting, population average presents the existence of abnormal elevation in its $3 \mathrm{D}$ visualization. It is due to the approach used by population average to forcedly replace blank data with a specific value obtained from statistic without comparing the value with the neighboring data. Meanwhile, neighbor average successfully prevents the presentation of abnormal elevation data since the approach used in this method is to absorb its neighboring elevation data. Thus, the comparison concludes that neighbor average outperform population average to produce $3 \mathrm{D}$ digital elevation model.

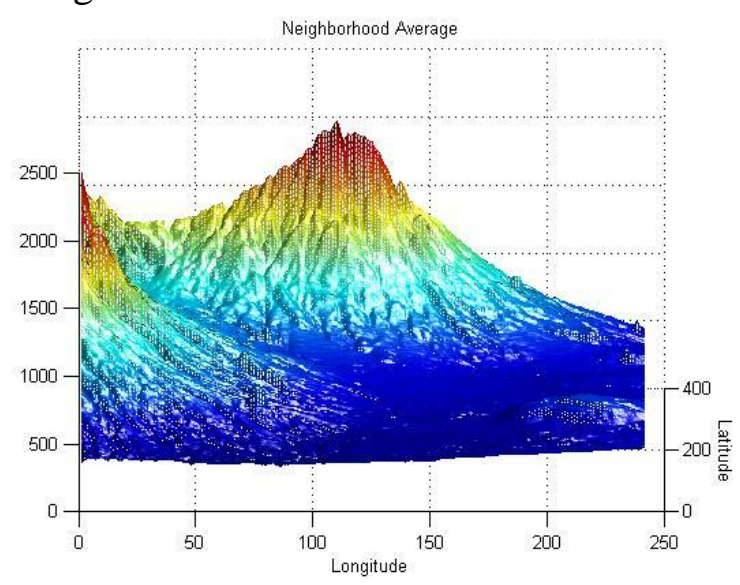

a. View angle $(0,20)$ 


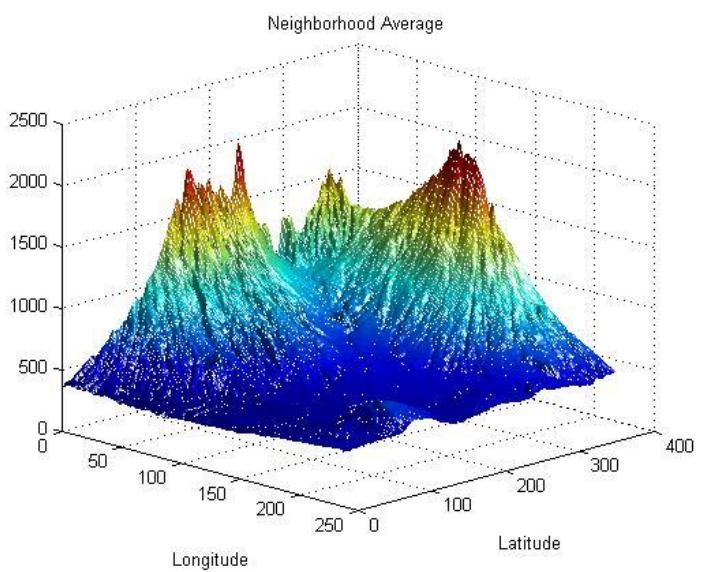

b. View angle $(45,20)$

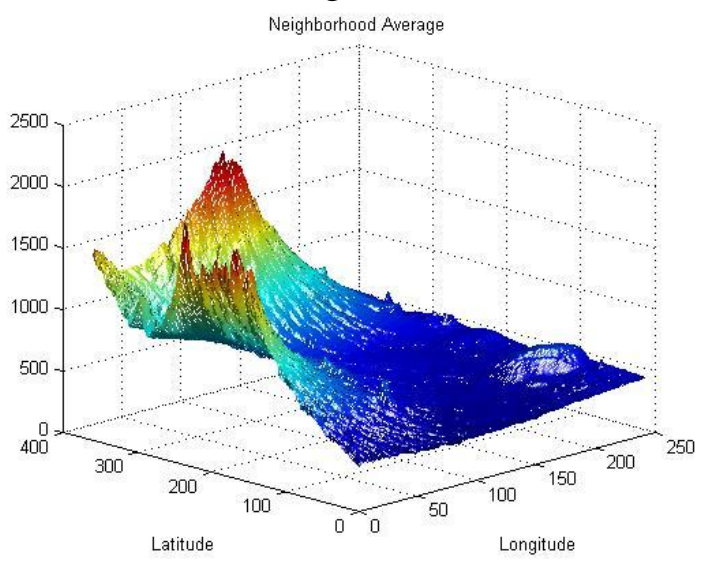

c. View angle $(-45,20)$

Figure 7. Enhanced visualization using different view angles

\section{REFERENCES}

[1] Miller, H.J., Wentz, E.A. (2003). Representation and Spatial Analysis in Geograhic Information Systems. Annals of the Association of American Geographers, 93(3), 2003, pp. 574-594.

[2] Kumar K, Pant MC, Satyal GS, Dumka RK. (2008). Comparison of Digital Surface Modeling Techniques for Sloping Hill Terrain using GPS Data. International Journal of Modeling and Simulation, 2008.

[3] Baltsavias EP, Favey E, Bauder A, Bosch H, and Pateraki M. (2001). Digital Surface Modeling by Airborne Laser Scanning and Digital Photogrammetry for Glacier Monitoring. Photogrammetric
Record, 17(98), pp. 243-273, October 2001.

[4] Nagai M, Shibasaki R, Kumagai H, Mizukami S, Manandhar D, and Zhao H. (2003). Construction of Digital Surface Model by Multisensor Integration from an Unmanned Helicopter. ISPRS WG V/6 - Visualization and Animation, 2003.

[5] Tollingerova D and Pavelka K. (2008). Creating of Digital Surface Model and Orthophoto from ASTER Satellite Data and Their Application in Land/Water Research. Soil and Water Res, 3, 2008 (2): 52-61.

[6] Meszaros M, Szatmari J, Tobal Z, and Mucsi L. (2008). Extraction of Digital Surface Models from Corona Satellite Stereo Images. Journal of Environmental Geography, Vol. I, No. 1-2, pp. 5-10.

[7] Shuttle Radar Topography Mission. Image Product Descriptions. Downloaded from www2.jpl.nasa.gov, 29 May 2009.

[8] Farr, TG, et al. (2007). The Shuttle Radar Topography Mission. Geophys, 45, RG2004, doi:10.1029/2005RG000183.

[9] Economic Division and Investment. (2006). General Description of Malang City. Downloaded from The Official Website of Local Government of Malang City, www.malangkota.go.id/investasi, 17 August 2009. 